\title{
Treatment with Angiotensin-(1-7) reduces inflammation in carotid atherosclerotic plaques
}

\author{
Rodrigo A. Fraga-Silva'; Silvia Q. Savergnini'; Fabrizio Montecucco ${ }^{2,3}$; Alessio Nencioni3 ${ }^{3}$ Irene Caffa ${ }^{3}$; Debora Soncini3; \\ Fabiana P. Costa-Fraga'; Frederico B. De Sousa'; Ruben D. Sinisterra4; Luciano A. S. Capettini'; Sébastien Lenglet²; Katia Galan²; \\ Graziano Pelli2; Maria Bertolotto ${ }^{3}$; Aldo Pende3 ${ }^{3}$; Giovanni Spinella5; Bianca Pane ${ }^{5}$; Franco Dallegri³; Domenico Palombo5; \\ François Mach ${ }^{2}$; Nikolaos Stergiopulos ${ }^{1}$; Robson A. S. Santos ${ }^{6 \# ;}$; Rafaela F. da Silva ${ }^{6 \#}$
}

${ }^{1}$ Institute of Bioengineering, Ecole Polytechnique Fédérale de Lausanne, Lausanne, Switzerland; 2Division of Cardiology, Faculty of Medicine, Foundation for Medical Researches, University of Geneva, Geneva, Switzerland; ${ }^{3}$ First Clinic of Internal Medicine, Department of Internal Medicine, University of Genoa School of Medicine, IRCCS Azienda Ospedaliera Universitaria San Martino-IST Istituto Nazionale per la Ricerca sul Cancro, Genoa, Italy; ${ }^{4}$ Department of Chemistry, Federal University of Minas Gerais, Brazil; ${ }^{5}$ Vascular and Endovascular Surgery Unit, Department of Surgery, San Martino Hospital, Genoa, Italy; ${ }^{6}$ Department of Physiology and Biophysics, Federal University of Minas Gerais, Brazil

\begin{abstract}
Summary
Angiotensin (Ang)-(1-7), acting through the receptor Mas, has atheroprotective effects; however, its role on plaque vulnerability has been poorly studied. Here, we investigated the expression of the renin-angiotensin system (RAS) components in stable and unstable human carotid plaques. In addition, we evaluated the effects of the chronic treatment with an oral formulation of Ang-(1-7) in a mouse model of shear stress-determined carotid atherosclerotic plaque. Upstream and downstream regions of internal carotid plaques were obtained from a recently published cohort of patients asymptomatic or symptomatic for ischaemic stroke. Angiotensinogen and renin genes were strongly expressed in the entire cohort, indicating an intense intraplaque modulation of the RAS. Intraplaque expression of the Mas receptor mRNA was increased in the downstream portion of asymptomatic patients as compared to corresponding region in symptomatic patients. Conversely, AT1 receptor gene expression was not modified between
\end{abstract}

asymptomatic and symptomatic patients. Treatment with Ang-(1-7) in $A \mathrm{Ap}^{-1-}$ mice was associated with increased intraplaque collagen content in the aortic root and low shear stress-induced carotid plaques, and a decreased MMP-9 content and neutrophil and macrophage infiltration. These beneficial effects were not observed in the oscillatory shear stress-induced plaque. In vitro incubation with Ang-(1-7) did not affect ICAM-1 expression and apoptosis on cultured endothelial cells. In conclusion, Mas receptor is up regulated in the downstream portions of human stable carotid plaques as compared to unstable lesions. Treatment with the oral formulation of Ang-(1-7) enhances a more stable phenotype in atherosclerotic plaques, depending on the local pattern of shear stress forces.

\section{Keywords}

Angiotensin, atherosclerosis, inflammation, plaque stability

Financial support:

This work was supported by the Brazilian Swiss Joint Research Program [590008/2010-2 to N.S, R.A.S.S., and F.M.]; Fundação de Amparo à Pesquisa do estado de Minas Gerais (FAPEMIG) [01/12 CBB APQ 01542/12 to R.F.S.]; Conselho Nacional de Desenvolvimento Científico e Tecnológico (CNPQ) [478065/2012-4 to R.F.S., 201603/2011-0 to R.A.FS.]; European Commission - Framework Programme for Research and Technological Development (EU FP7) [3201668 to F. Mach]; Swiss National Science Foundation Grants [\#310030-118245 to F. Mach, \#32002B-134963/1 to F. Montecucco]; Novartis Consumer Health [to R.A.FS] and Italian Ministry of Health [GR-2008-1135635 to A. Nencioni]. Received: June 3, 2013

Accepted after major revision: November 11, 2013

Prepublished online: February 6, 2014

doi:10.1160/TH13-06-0448

Thromb Haemost 2014; 111: 736-747

\section{Introduction}

Atherosclerosis remains one of the leading causes of mortality in developed and developing nations (1). This elevated complication rate is mainly due to the conversion of a stable atherosclerotic plaque into an unstable plaque. These vulnerable plaques are susceptible to rupture, having dramatic consequences including acute ischaemic stroke and myocardial infarction $(2,3)$. In addition to systemic and enviromental risk factors, the development of athero- sclerotic plaques occurs preferentially at vessel bifurcation and curved areas of the vasculature due to the presence of low endothelial shear stress force acting locally on the arterial wall $(4,5)$. Recently, local low endothelial shear stress has been identified as a biomechanical determinant increasing plaque vulnerability (6). However, the molecular mechanisms undelying plaque vulnerability remain largely unknown.

The renin-angiotensin system (RAS) is known to be involved in the initiation, progression and vulnerability of the atherosclerotic 
plaque (7-10). There are clear evidences that angiotensin (Ang) II, synthesised by the catalytic activity of angiotensin converting enzyme (ACE),might influence atherogenesis through the increase in vascular permeability, leukocyte infiltration and low-density lipoprotein (LDL) oxidation and uptake (11-13). On the other hand, this peptide has been also shown to increase plaque vulnerability via direct modulation on macrophage trapping, oxidative stress, and matrix metalloproteinase activation (10, 14-17).

Currently, the new counter-regulatory mediator Ang-(1-7) in the RAS has been well established (18-21). This molecule is primarily produced from Ang II by the catalytic activity of angiotensin converting enzyme 2 (ACE2) $(20,21)$. Its binding to Mas receptor (22), often promotes opposite actions to Ang II $(18,21)$. In fact, while Ang II is considered as a potent vasoconstrictor and pro-atherosclerotic mediator (7), Ang-(1-7) has been thought to induce vasoprotective and atheroprotective effects (23-25).

In the present study, we evaluated the expression of RAS components in different regions of carotid plaques from patients with severe carotid stenosis (symptomatic or asymptomatic for ischaemic stroke). Moreover, we investigated the potential protective effects of an oral formulation of Ang-(1-7) included in hydroxypropyl- $\beta$-cyclodextrin [Ang-(1-7)-CyD] $(26,27)$, on intraplaque histological parameters associated with vulnerability in a mouse model of shear stress-induced atherogenesis in carotid artery.

\section{Methods}

\section{Atherosclerotic human samples and study design}

Residual samples form upstream and downstream regions (considering the blood flow) of internal carotid plaques obtained from patients with high-grade internal carotid stenosis ( $>70 \%$ luminal narrowing) and asymptomatic $(\mathrm{n}=63)$ or symptomatic $(\mathrm{n}=18)$ for ischaemic stroke were analysed in this sub-study. The entire human cohort, belonging to the previously published unmatched case-control study (performed in 2008-2009 at San Martino Hospital, Genoa, Italy), was enrolled in this sub-study (28). No significant difference in terms of age, sex, co-morbidities, and laboratory parameters was found between asymptomatic and symptomatic patients (28).

Patients were classified as symptomatic for ischaemic stroke after the first episode of ipsilateral ischaemic stroke (focal neurologic deficit of acute onset lasting more than 24 hours (h) occurring between 30 and 10 days prior to endarterectomy). They were defined as asymptomatic when they had no history of ischaemic symptoms and in the absence of signs of cerebral necrosis at magnetic resonance imaging [MRI] with diffusion sequences. Both asymptomatic and symptomatic patients underwent carotid endarterectomy (CEA) according to the recommendations published by the Asymptomatic Carotid Surgery Trial (ACST), the European Carotid Surgery Trial (ECST) and the North American Symptomatic Carotid Endarterectomy Trial (NASCET) (29-31). Exclusion criteria were: spontaneous cerebral embolism during 30 minutes (min) preoperatively and during the dissection phase of the operation, malignant hypertension, acute coronary artery disease, any cardiac arrhythmias, congestive heart failure (II, III and IV NHYA classes), liver or renal disorders or functional abnormalities, acute and chronic infectious diseases, autoimmune and rheumatic diseases, cancer, endocrine diseases, inflammatory bowel diseases and anti-inflammatory (other than aspirin) medications, oral anticoagulant treatments, hormone, cytokine or growth factor therapies (28). After surgical incision, upstream and downstream portions (considering the blood flow) of human internal carotid plaque were quickly processed as previously described (28). The segments were snap-frozen in liquid nitrogen and stored at $-80^{\circ} \mathrm{C}$ for mRNA isolation and measurements. The Medical Ethics Committee of San Martino Hospital approved the study and participants provided written informed consent. The study was conducted in compliance with the Declaration of Helsinki.

\section{Animal model}

To induce a vulnerable atherosclerotic plaque we used the mouse model of shear stress-induced atherogenesis and plaque vulnerability developed by the research group of Prof. R. Krams (4). In this model, specific patterns of shear stress are applied to the wall of the carotid artery of apolipoprotein $\mathrm{E}$ deficient $\left(\mathrm{ApoE}^{-/-}\right)$mice. The composition of atherosclerotic plaques is determined by the distinct patterns of shear stress. Plaques induced by low shear stress have histological features of vulnerable plaques, whereas plaques developed by vortexes of oscillatory shear stress display a more stable phenotype $(4,32,33)$.

$\mathrm{ApoE}^{-/-}$mice in a $\mathrm{C} 57 \mathrm{BL} / 6 \mathrm{~J}$ background were obtained from Jackson Laboratories. Animals (15-20 weeks of age) were fed for 11 weeks of the experimental period with a Western-type diet (15\% cocoa butter and $0.25 \%$ cholesterol, Diet W; abDiets, Netherlands). After a two-weeks period of Western diet, specific patterns of shear stress were applied to the right carotid artery by the placement of a cast around the vessel (4). The cast consists of two longitudinal halves of a cylinder with a cone-shaped lumen. The geometry of the cast was developed to produce vortices downstream, therefore exposing the artery wall to oscillations in shear stress (OSS) in the downstream region and a low shear stress (LSS) in the region upstream the blood flow.

The surgical procedure of cast implantation was performed as previously described (32). Briefly, the animals were anesthetised by $2-3 \%$ isoflurane inhalation, and the anterior cervical triangles were accessed by a sagittal anterior neck incision. Both halves of the cast were placed around the right common carotid artery and fixed with a suture. During surgery, adequacy of anesthesia was monitored by careful visual and tactile control of mouse consciousness (changes in breathing rate and volume, heart rate, sweating and tearing). After closing the wounds, the animals were allowed to recover. Nine weeks after surgery, the animals were euthanised to collect tissue and serum samples. During the last three weeks before euthanizing (from week 6 to 9 of cast implantation), mice were orally treated with Ang-(1-7)-CyD (equivalent of $30 \mu \mathrm{g} / \mathrm{kg} /$ day of Ang-(1-7) for five consecutive days per week given by gavage) (27) or respective vehicle control (hydroxypropyl $\beta$-cyclodextrin). Then, mice were euthanised (ketamine $100 \mathrm{mg} / \mathrm{kg}$, xylazine 


\begin{tabular}{|c|c|c|c|c|}
\hline Gene & Function & Nucleotide sequence & Size (bp) & Accession number \\
\hline \multirow[t]{3}{*}{ AGT } & Fw & $5^{\prime}$ - aactatctccccggaccatc $-3^{\prime}$ & \multirow[t]{3}{*}{117} & \multirow[t]{3}{*}{ NM_000029 } \\
\hline & Rv & $5^{\prime}$ - tcagctcggtgtgcagaat $-3^{\prime}$ & & \\
\hline & probe & $5^{\prime}-$ FAM-acctgaccatgccccaactggt-BHQ1 -3' & & \\
\hline \multirow[t]{3}{*}{ AT1 } & Fw & $5^{\prime}$ - cgcgggtttgatatttgaca $-3^{\prime}$ & \multirow[t]{3}{*}{132} & \multirow[t]{3}{*}{ NM_000685 } \\
\hline & Rv & $5^{\prime}$ - atcacctgggtcgaatttgtt $-3^{\prime}$ & & \\
\hline & probe & 5'- FAM-tgatctaaaatggctgggtttttatctgaa-BHQ1 -3' & & \\
\hline \multirow[t]{3}{*}{ ACE } & Fw & $5^{\prime}$ - ctaagcggcagcagtacaac $-3^{\prime}$ & \multirow[t]{3}{*}{94} & \multirow[t]{3}{*}{ NM_000789 } \\
\hline & Rv & $5^{\prime}$ - cagcaggtggcagtcttg $t-3^{\prime}$ & & \\
\hline & probe & 5'- FAM-agcaggatctactccaccgccaag-BHQ1 - $3^{\prime}$ & & \\
\hline \multirow[t]{3}{*}{ ACE2 } & Fw & $5^{\prime}-$ tgcctatgtgagggcaaag $-3^{\prime}$ & \multirow[t]{3}{*}{92} & \multirow[t]{3}{*}{ NM_021804 } \\
\hline & Rv & $5^{\prime}$ - cccacatatcaccaagcaaa -3' & & \\
\hline & probe & 5'- FAM-tgatgaatgcctatccttcctatatcagtcc-BHQ1 -3' & & \\
\hline \multirow[t]{3}{*}{ MAS } & Fw & $5^{\prime}$ - gaacccacgaacatctcaact $-3^{\prime}$ & \multirow[t]{3}{*}{140} & \multirow[t]{3}{*}{ NM_002377 } \\
\hline & Rv & $5^{\prime}$ - aagcacaggaaccagaggag $-3^{\prime}$ & & \\
\hline & probe & 5'- FAM-acgcctcagtcgggaatgcac-BHQ1 -3' & & \\
\hline \multirow[t]{3}{*}{ REN } & Fw & $5^{\prime}$ - ggctgt ttgattatgtcgtgaag $-3^{\prime}$ & \multirow[t]{3}{*}{79} & \multirow[t]{3}{*}{ NM_000537 } \\
\hline & Rv & $5^{\prime}$ - tattctttgcctcccaggtg $-3^{\prime}$ & & \\
\hline & probe & 5'- FAM-ccctacactccccgacatctctttc-BHQ1 -3' & & \\
\hline \multirow[t]{3}{*}{ RPS13 } & Fw & $5^{\prime}$-cgtccccacttggttgaag- $3^{\prime}$ & \multirow[t]{3}{*}{90} & \multirow[t]{3}{*}{ NM_001017 } \\
\hline & Rv & $5^{\prime}$-ccgatctgtgaaggagtaagg-3' & & \\
\hline & probe & 5'- FAM-acatctgacgacgtgaaggagcagatt-BHQ1 -3' & & \\
\hline
\end{tabular}

Table 1: Human primers and probes used for real-time PCR.
$10 \mathrm{mg} / \mathrm{kg}$ ) and blood/tissue samples were collected. Serum glucose, triglycerides, total cholesterol, LDL cholesterol and highdensity lipoprotein (HDL) cholesterol were routinely measured and expressed as mmol/l. After collection, the tissues were removed and snap-frozen in liquid nitrogen and stored at $-80^{\circ} \mathrm{C}$ for protein measurements or frozen in cryoembedding medium for histological analysis. This animal study was approved by local ethics committee and Swiss Regulatory Authorities (authorisation number 2026.2) and it is according with to the Guide for the Care and Use of Laboratory Animals of United States National Institutes of Health.

\section{Real-time RT-PCR}

Total mRNA was isolated with Tri-reagent (MRC Inc.) from upstream or downstream specimens of human carotid plaques. Reverse transcription was performed using the ImProm-II Reverse Transcription System (Promega, Madison, WI, USA) according to the manufacturer's instructions. Real-time PCR (StepOne Plus, Applied Biosystems, Foster City, CA, USA) was performed with the ABsolute ${ }^{\mathrm{m}}$ QPCR Mix (ABgene, Epsom, UK).

Specific primers and probes ( Table 1) were used to determine the mRNA expression of renin, angiotensinogen (AGT), ACE,
ACE2, Mas and AT1. The fold change of mRNA levels was calculated by the comparative $C_{t}$ method. The measured $C_{t}$ values were first normalised to the RPS13 internal control, by calculating a delta $C_{t}\left(\Delta C_{t}\right)$. This was achieved by subtracting the RPS13 $C_{t}$ values from the gene of interest $C_{t}$ value. A delta delta $\mathrm{Ct}\left(\Delta \Delta \mathrm{C}_{\mathrm{t}}\right)$ was calculated by subtracting the designated baseline control group $\Delta \mathrm{C}_{\mathrm{t}}$ value from the study group $\Delta \mathrm{C}_{\mathrm{t}}$ values. The $\Delta \Delta \mathrm{C}_{\mathrm{t}}$ was then plotted as a relative fold change with the following formula: $2^{-\Delta \Delta C t}$.

\section{Oil Red 0 staining}

Eleven sections per mouse carotid LSS and OSS and five sections per mouse aortic sinus were stained with Oil Red O, as previously described (34). Sections from carotid and aorta were counterstained with Mayer's hemalun and rinsed in distilled water. Quantifications were performed with MetaMorph software. Data were calculated as ratio of stained area on total lesion area.

\section{Immunohistochemistry in mouse carotid plaques and mouse aortic sinus}

Mouse carotid plaque samples processing were performed as previously described (32). Briefly, the carotid artery was macroscopi- 


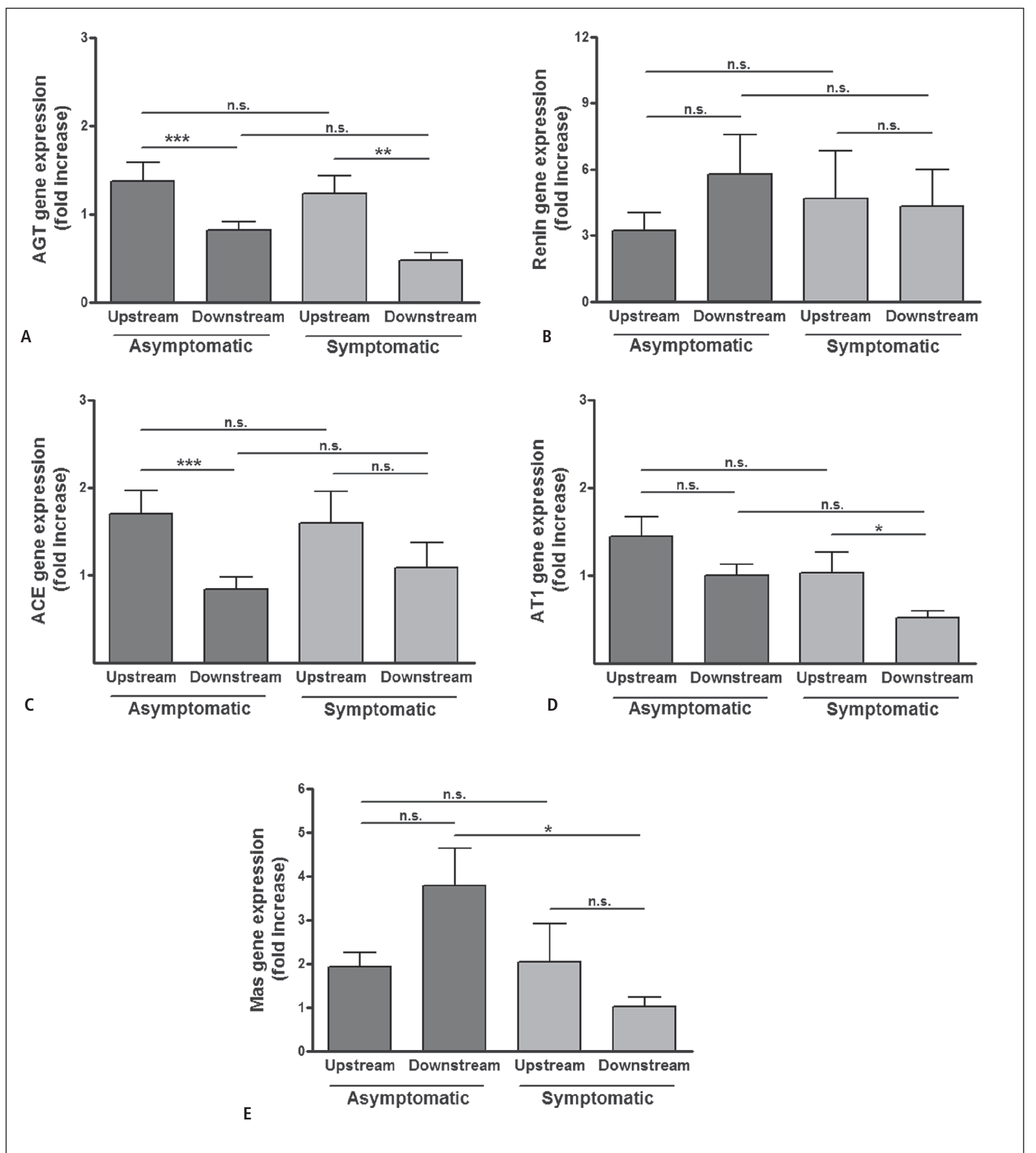

Figure 1: Expression of the Mas receptor gene is increased in the human stable plaques. Intraplaque mRNA expression of AGT (A), renin (B), ACE (C), AT1 receptor (D) and Mas receptor (E) in patients asymptomatic or symptomatic for ischaemic stroke. Relative expression normalised to house- keeping gene was calculated with the comparative $\mathrm{Ct}$ method and shown as fold change of mRNA levels. n.s.: non-significant, ${ }^{*} p<0.05,{ }^{* *} p<0.01$ and ${ }^{* * *} p<0.001$. Data were expressed as mean \pm SEM (asymptomatic patients: $n=63$; symptomatic patient: $n=18$ ). 
Table 2: Mouse serum lipid profile at sacrifice.

\begin{tabular}{l|l|l|l}
\hline $\begin{array}{l}\text { Serum lipid } \\
\text { profile } \\
(\mathbf{m m o l} / \mathrm{l})\end{array}$ & $\begin{array}{l}\text { Vehicle- } \\
\text { treated mice } \\
(\mathbf{n}=\mathbf{1 5})\end{array}$ & $\begin{array}{l}\text { Ang-(1-7)-CyD- } \\
\text { treated mice } \\
(\mathbf{n}=\mathbf{1 5})\end{array}$ & P-value \\
\hline Total Cholesterol & $23.57 \pm 1.84$ & $23.44 \pm 1.10$ & 0.9496 \\
\hline LDL $^{*}$-cholesterol & $20.30 \pm 1.63$ & $20.24 \pm 1.08$ & 0.9746 \\
\hline HDL+-cholesterol & $4.22 \pm 0.29$ & $4.09 \pm 0.18$ & 0.7075 \\
\hline Triglycerides & $0.86 \pm 0.06$ & $0.90 \pm 0.07$ & 0.7119 \\
\hline Fatty Free Acid & $0.72 \pm 0.09$ & $0.68 \pm 0.06$ & 0.7223 \\
\hline
\end{tabular}

Data are expressed as mean \pm SEM. P-value calculated according to unpaired t-test. *LDL: low-density lipoprotein. +HDL: high-density lipoprotein.

cally cut in the upstream and downstream regions of the cast device (LSS and OSS regions, respectively), and then frozen in OCT medium. LSS and OSS portions from all mice were serially cut in 5 $\mu \mathrm{m}$ transversal sections. Eleven sections per staining (separated by $45 \mu \mathrm{m}$ from each other) were used. Transversal sections of $5 \mu \mathrm{m}$ of the aortic sinus were serially cut in through the heart and aortic roots. For each aortic sinus, five sections (separated by $50 \mu \mathrm{m}$ from each other) were assessed for each staining. The sections were fixed in acetone and immunostained with specific anti-mouse CD68 (macrophages, dilution: 1:400; ABD Serotec, Düsseldorf, Germany), anti-mouse Ly-6B.2 (neutrophils, dilution: 1:50; ABD Serotec), and anti-mouse MMP-9 (dilution: 1:60; R\&D Systems, Abigdon, UK). Quantifications were performed with MetaMorph software. Data were calculated as percentages of stained area on total lesion area (macrophages and MMP-9) or number of infiltrating cells on $\mathrm{mm}^{2}$ of lesion area (neutrophils).

\section{Sirius Red staining}

Sections of mouse carotid LSS and OSS, and aortic sinus were rinsed with water and incubated with $0.1 \%$ Sirius red (Sigma Chemical Co, St Louis, MO, USA) in saturated picric acid for 90 $\mathrm{min}$. Sections were rinsed twice with $5 \%$ acetic acid in water for 10 seconds (sec), then immersed in absolute ethanol three times before clearing in xylene twice and cover-slipping. The sections were then photographed with identical exposure settings under ordinary polychromatic light microscopy. Total collagen quantifications were performed with MetaMorph software. Data were calculated as percentages of stained area on total lesion area.

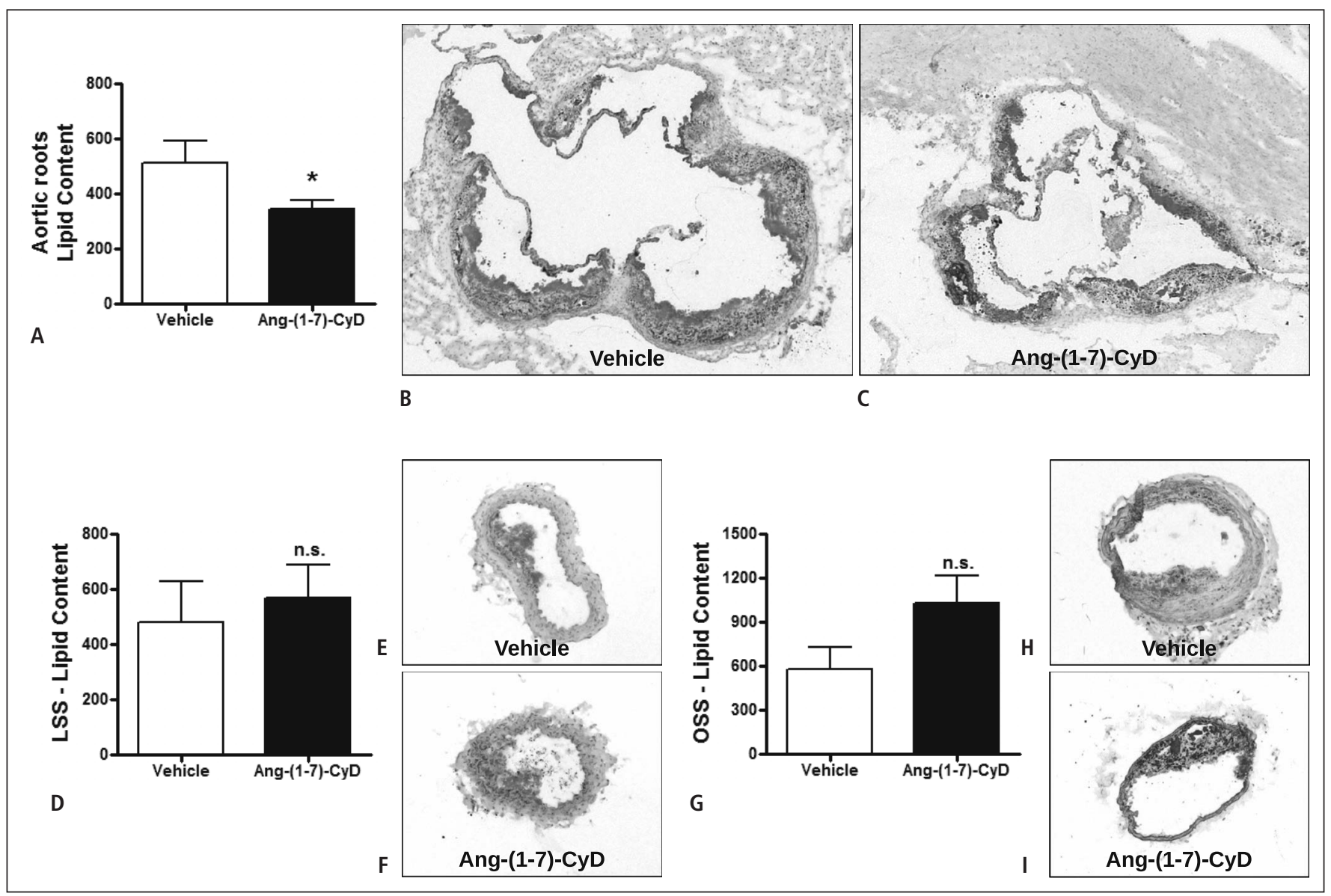

Figure 2: Treatment with Ang-(1-7)-CyD reduced the lipid content in aortic root plaques, but not in plaques developed at both LSS and OSS regions. Quantification and representative microphotographs lipid staining in: mouse aortic root (A-C), carotid LSS region (D-F) and carotid OSS regions (G-I). n.s.: non-significant, ${ }^{*} p<0.05$. Data were expressed as mean \pm SEM ( $n=15$ per group). 


\section{Human vascular endothelial cells (HECV) culture and flow cytometry analysis of ICAM-1}

HECV (1.6 x $10^{4}$ cells/well) were plated in six-well plates and allowed to adhere overnight. Cells were then treated with control medium (CTL) or Ang-(1-7) (at $10^{-8}$ to $\left.10^{-6} \mathrm{M}\right)(27,35,36)$ in the presence or absence of $20 \mathrm{ng} / \mathrm{ml}$ of human recombinant TNFalpha (R\&D Systems Ltd) for $3 \mathrm{~h}$. Floating and adhering cells were collected from culture wells by repeatedly washing and by trypsinisation. Subsequently, cells were stained with propidium iodide ([PI], Sigma Aldrich) to determine cells viability, or anti-human ICAM-1/CD54-Phycoerythrin (Pharmingen, San Jose, CA, USA) accordingly to the manufacturer's instructions. The samples were analysed by flow cytometry with a FACS Calibur (Becton Dickinson, San Diego, CA, USA). Ten thousand events for each sample were acquired. Results were expressed as mean fluorescence intensity.

\section{Data analysis}

Mann-Whitney U nonparametric test was used for comparisons between mRNA expression within human plaques (asymptomatic vs symptomatic and upstream vs downstream), between parameters of mouse plaque vulnerability in Ang-(1-7)- and vehicletreated mice. One-way ANOVA was used for multiple group comparison, and the unpaired Student t-test was used for two group comparison for in vitro results. Statistical analysis was performed using GraphPad Prism software (GraphPad, San Diego, CA, USA). A value of $p<0.05$ was considered significant and the results are expressed as mean \pm SEM.

\section{Results}

\section{RAS components are differently expressed in human carotid plaques}

We observed that the AGT gene, the precursor of the RAS cascade, was highly expressed in the entire cohort, indicating a potential intraplaque modulation of RAS. However, no difference in the expression of AGT gene between the asymptomatic and symptomatic patients was shown ( Figure 1A). Nevertheless, AGT expression was reduced in the downstream regions as compared to upstream portions in both asymptomatic and symptomatic patients ( Figure 1A). Renin mRNA was also highly expressed in human plaques. Anyway, no significant differences were observed between the asymptomatic and symptomatic patients, or between the upstream and downstream plaque portions ( Figure 1B).

Considering the intraplaque mRNA expression of ACE and AT1 receptor, no difference was observed between asymptomatic and symptomatic patients. However, ACE mRNA levels were decreased in the downstream portions of asymptomatic carotid plaques as compared to upstream ( Figure 1C). On the other hand, AT1 receptor mRNA was reduced in the downstream portions of symptomatic plaques as compared to upstream ( $>$ Figure
1D). The mRNA expression of ACE2 was very weak in all human plaques, being detected only in few samples with no significant differences among the groups (data not shown). Conversely, the Mas receptor mRNA was highly expressed within human carotid plaques. In particular, the expression of this receptor was significantly increased in the downstream portions of the asymptomatic patients as compared to the corresponding regions of the symptomatic patients ( Figure 1E). No statistical differences were observed between upstream and downstream regions in both asymptomatic and symptomatic patients ( $\triangleright$ Figure $1 \mathrm{E}$ ).

\section{Treatment with Ang-(1-7)-CyD improves histological parameters of intraplaque vulnerability}

Since the expression of Mas was increased in the downstream portions of human more stable plaques (asymptomatic patients), we sought to investigate the potential benefits of a three-week oral treatment with the Ang-(1-7)-CyD as compared to the corresponding vehicle $(\mathrm{CyD})$ on mouse aortic roots and carotid plaque vulnerability and serum lipid profile. Treatment with Ang-(1-7)CyD did not affect serum lipid profile as compared to control vehicle ( Table 2). Conversely, treatment with Ang-(1-7)-CyD reduced the lipid content within aortic root plaques $(>$ Figure $2 \mathrm{~A}-\mathrm{C})$. However, no change on lipid deposition was observed in both LSS ( Figure 2D-F) and OSS regions ( $\triangleright$ Figure 2G-I). Interestingly, treatment with Ang-(1-7)-CyD markedly increased the collagen content in the aortic root ( Figure $3 \mathrm{~A}-\mathrm{C}$ ) and LSSinduced plaques ( Figure 3G-I), but not in the OSS-induced plaques ( Figure 3J-M) as compared to control vehicle. Accordingly, MMP-9 intraplaque content was decreased in aortic roots $(>$ Figure $3 \mathrm{D}-\mathrm{F})$ and LSS-induced plaques $(>$ Figure $3 \mathrm{~N}-\mathrm{P}$ ) of Ang-(1-7)-CyD-treated mice, but not within OSS-induced plaques (- Figure 3Q-S).

Further, we investigated the effect the Ang-(1-7)-CyD treatment on intraplaque infiltration of inflammatory cells. Treatment with Ang-(1-7)-CyD markedly reduced the accumulation of macrophages and neutrophils within aortic root plaques ( $\$$ Figure $4 \mathrm{~A}-\mathrm{C}$ and D-F, respectively) as compared to control vehicle. In plaques induced by LSS, the Ang-(1-7)-CyD treatment reduced neutrophil ( Figure $4 \mathrm{~N}-\mathrm{P}$ ), but not macrophage infiltration ( $\triangleright$ Figure 4G-I). Treatment with Ang-(1-7)-CyD did not modify the intraplaque infiltration of these inflammatory cells in OSS regions ( $>$ Figure 4J-M and Q-R).

\section{Treatment with Ang-(1-7) does not affect ICAM-1 expression on HECV in vitro}

As expected, TNF-alpha significantly increased the expression of ICAM-1 on HECV. Co-incubation with Ang-(1-7) did not affect ICAM-1 upregulation induced by TNF-alpha ( $>$ Figure $5 \mathrm{~A}$ ). In the absence of TNF-alpha, Ang-(1-7) did not produce any effect on ICAM-1 expression on HECV ( Figure 5A). Importantly, treatment with Ang-(1-7) did not induce any significant effect on HECV apoptosis ( Figure 5B). 


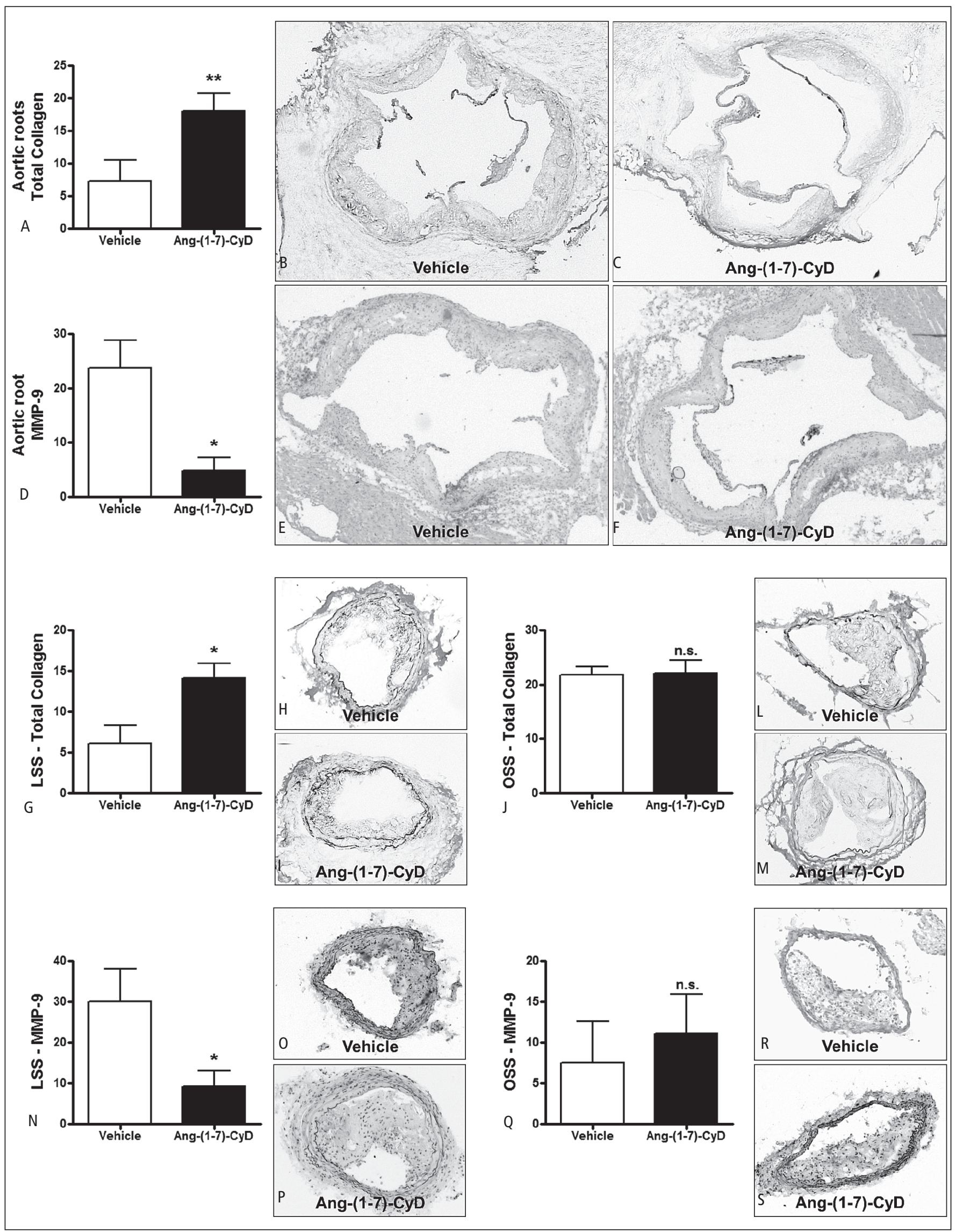




\section{Discussion}

\section{Human study}

It is largely known that the "systemic" RAS is involved in the initiation, progression and vulnerability of atherosclerotic plaques (7, 8). In the present study, we observed that AGT and renin, the precursor substrate and the primary enzyme, respectively, from the RAS peptidergic cascade, were highly expressed locally within human plaques. These data suggest an intense modulation of the RAS components within the inflamed atherosclerotic tissue via potential paracrine and/or autocrine actions. Interestingly, we found that the expression of the AGT, ACE and AT1 receptor genes was up regulated in the upstream regions of the plaques as compared to the downstream, indicating that the RAS might be differentially influenced by the distinct patterns of shear stress. In line with these results, a recent study showed that oscillatory flow induces a time-dependent increase in the expression of the AT1 receptor on endothelial cells exposed to a laminar flow (37). In the same study, in vivo data showed that the expression of the AT1 receptor was increased on the endothelial cells lining on the inner curvature of mouse aortic arch (area associated with the initiation of atherosclerosis), but it was almost absent on the outer curvatures (37). This study mainly addressed the early changes induced by wall shear stress on isolated endothelial cells and on the endothelium of plaque-prone area. To our knowledge, our work is the first to address the expression of RAS components on human atherosclerotic plaques associated to different pattern of shear stress. At present, our data reveal the expression of the RAS genes in the whole plaque biopsies and therefore the cellular source of each component is not yet known. Considering that no additional histological material was available from our human cohort, we could not address this point with co-localisation assays and, thus, this point represents an important limitation of our study.

While the involvement of the Ang II/AT1R axis in the occurrence of atherosclerotic plaque instability has already been addressed (8), less is known about the ACE2/Ang-(1-7)/Mas receptor axis. The ACE2 is reported as the major enzyme responsible for Ang-(1-7) formation (38, 39). However, this peptide might be generated by additional pathways involving prolylcarboxypeptidase, prolylendopeptidase or neutral endopeptidase (20). Campbell et al. reported that in human coronary vessels Ang-(1-7) is mainly formed by prolylendopeptidase (40), indicating that the main pathway of Ang-(1-7) formation may vary depending on the vascular bed. In our study, we detected a very weak expression level of the ACE2 gene in human plaques. The mRNA was detected in

\footnotetext{
Figure 3: Ang-(1-7)-CyD increased the collagen content and decreased MMP-9 expression in aortic root and LSS-induced plaques. Quantification and representative microphotographs of collagen staining in: mouse aortic root (A-C), carotid LSS region (G-I) and carotid OSS regions (J-M). Quantification and representative microphotographs of MMP-9 immunostaining in: mouse aortic root (D-F), carotid LSS region (N-P) and carotid OSS regions (Q-S). ${ }^{*} p<0.05$ and ${ }^{* *} p<0.01$. Data were expressed as mean \pm SEM ( $n=15$ per group).
}

only few samples, with no significant difference between groups. However, due to its multiple pathways of formation, it was not possible to estimate the local level of Ang-(1-7). Differently from our results, Sluimer et al. showed that ACE2 mRNA is expressed in both early and advanced human carotid atherosclerotic lesions (41). In their work, the quantification of immunoreactivity analysis for ACE2 showed no significant differences during all stages of atherosclerosis. Therefore, also this aspect requires further confirmation.

Ang-(1-7) acting through Mas receptor often promotes opposite effects to the Ang II, thereby promoting many beneficial cardiovascular outcomes (18-21, 42). In our study, we found that the expression of the Mas receptor mRNA was very high in all plaque, with a significant increase in the downstream portion of asymptomatic patients as compared to corresponding regions of symptomatic patients. We have previously shown that plaques from the asymptomatic patients exhibited a more stable phenotype when compared to the plaques from the symptomatic patients (32). Thus, the increased expression of Mas receptor in the downstream portion of asymptomatic patients may suggest a potential protective role of this receptor on plaque vulnerability. To better investigate this potential role, we treated $\mathrm{ApoE}^{-/-}$mice submitted to carotid low shear stress-induced vulnerable plaques, with a novel oral formulation of Ang-(1-7) that has been shown to potently activate the Mas receptor $(26,27)$.

\section{Animal study}

The importance of wall shear stress to the etiology of atherosclerosis is known since more than two decades (43). However, only recently it was developed an appropriate animal model to study the cause-effect relationship between the patterns of wall shear stress and plaque formation (4). The cast model of shear stress-induced atherogenesis and plaque vulnerability described by Cheng et al. (4) has a further advantage that it allows the induction of plaques with distinct phenotypes in the same carotid artery. While low shear stress (LSS) fields promote the formation of larger plaques with a more vulnerable phenotype, oscillatory shear stress (OSS) induces more stable plaques $(4,32)$. More recently, data from this model and also from other human studies $(6,32,44)$ identified LSS as a key biomechanical mediator in the conversion of atherosclerotic lesion from stable to unstable plaque. By using this animal model, we observed that Ang-(1-7)-CyD increased the stability of LSS-induced plaques, but not OSS plaques, suggesting that the Ang-(1-7) treatment inhibits the conversion of stable plaques to vulnerable plaques by OSS.

The potential atheroprotective effect of the Ang-(1-7) was demonstrated for the first time in 2010 (23). Tesanovic et al. showed that the long-term infusion of the Ang-(1-7) promoted a reduction on plaque size and improved the vascular endothelial function of ApoE $\mathrm{E}^{-/}$mice (23). They suggested that these effects were mediated by an increase in the expression of eNOS and by an improvement on vascular oxidative stress, involving a complex interaction of both Mas and AT2 receptors (23). In line with these results, AVE-0991, a non-peptide synthetic Mas agonist, inhibited athero- 


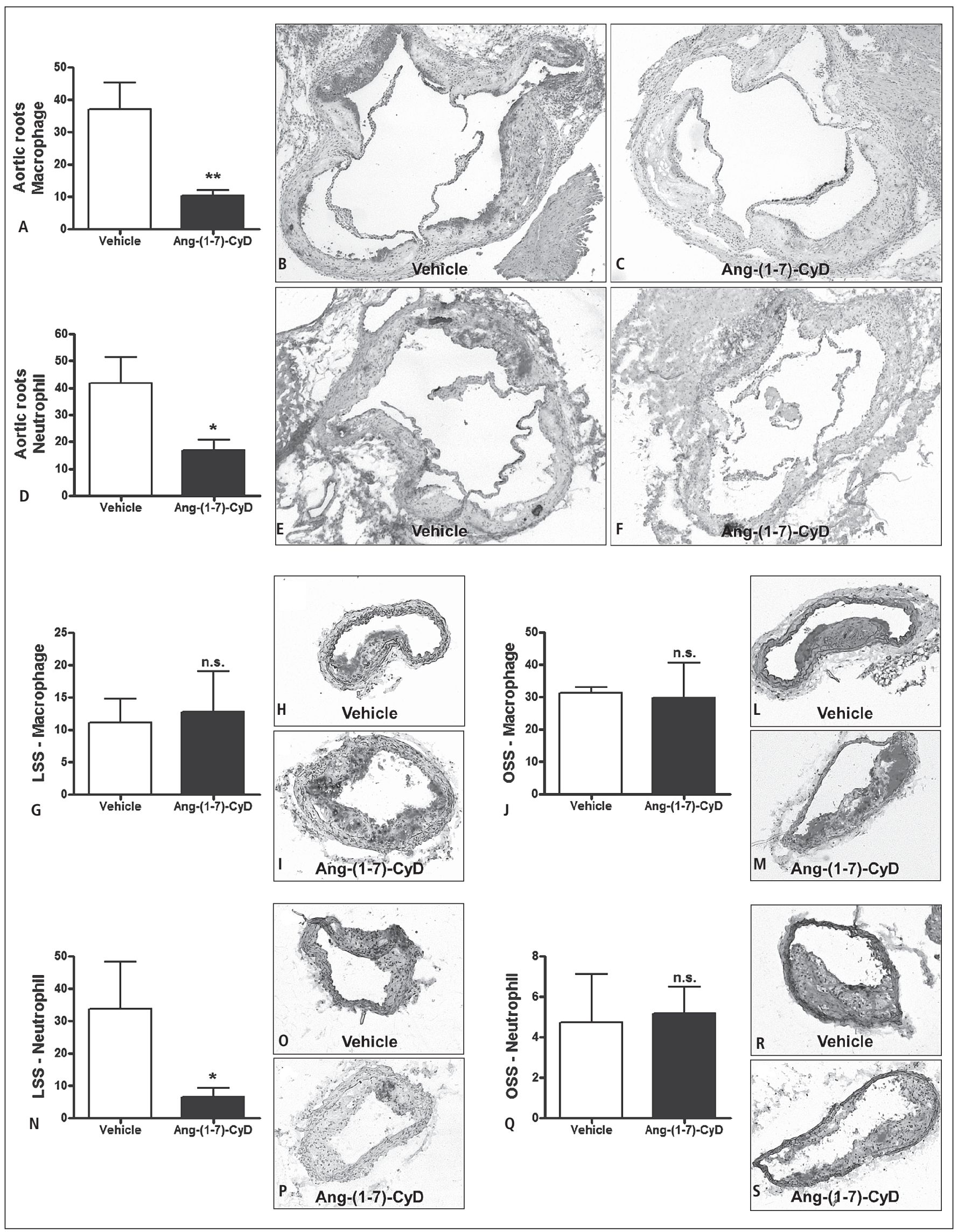


genesis in $\mathrm{ApoE}^{-/-}$mice, which was blocked by the selective Mas antagonist A-779, evidencing the protective effect of Mas receptor (24). In both studies, there were no data available regarding the potential protective role of Ang-(1-7) on the plaque phenotype. Here, we observed that the Ang-(1-7)-CyD treatment not only reduced lipid content in the aortic root plaques, but also increased collagen content, decreased MMP-9 expression and reduced neutrophil and macrophage infiltration, resulting in plaques with a more stable phenotype. The Ang-(1-7)-CyD treatment was also effective on carotid plaques. However, as plaque stabilisation occurred exclusively in plaques developed by LSS, but not by OSS, we can hypothesise that still unknown haemodynamic dependentmechanisms might influence Ang-(1-7) efficacy. In this line, a previous work has shown that overexpression of ACE2, the precursor enzyme of Ang-(1-7) peptide, stabilises atherosclerotic plaque in aorta from rabbits, by reducing macrophage infiltration and lipid deposition and by increasing collagen content (45). However, this work did not clarify the distinct the roles of Ang II and Ang-(1-7) in the beneficial actions of ACE2 overexpression, since this enzyme increases the Ang-(1-7) levels, but concomitantly decreases the levels of Ang II.

Of note, in order to clarify the cellular mechanism by which Ang-(1-7) reduced the inflammatory cell infiltration within atherosclerotic plaques, we attempted to evaluate the effects of the peptide on adhesion molecule expression on cultured HECV. We observed that co-incubation with Ang-(1-7) did not change ICAM-1 expression on both untreated and TNF-alpha-stimulated HECV, suggesting that the protective action of this peptide on atherosclerotic plaque phenotype might be not related to reduction on adhesion molecule expression on endothelial cells. Importantly, treatment with Ang-(1-7) did not induce any effect on HECV apoptosis, suggesting that doses selected for these experiments were not toxic for HECV.

\section{Clinical perspectives and conclusions}

The present study shows that the RAS components are differently expressed in atherosclerotic plaques associated with distinct shear stress patterns. Importantly, the Mas receptor is upregulated in downstream portions of human stable plaques, suggesting a protective effect of this receptor against plaque vulnerability. An oral chronic treatment with the Ang-(1-7)-CyD (a Mas receptor agonist) in $\mathrm{ApoE}^{-/-}$mice enhances a stable phenotype in atherosclerotic plaques depending on the local patterns of shear stress.

The Ang-(1-7)-CyD formulation used here, not only allows the administration of the Ang-(1-7) orally, but also promotes a sus-

Figure 4: Treatment with Ang-(1-7)-CyD reduced the accumulation of macrophages and neutrophils in the vulnerable plaques. Quantification and representative microphotographs of staining for macrophages in: mouse aortic root (A-C), carotid LSS region (G-I) and carotid OSS regions (J-M). Quantification and representative microphotographs of staining for neutrophils in: mouse aortic root (D-F), carotid LSS region (N-P) and carotid OSS regions $(\mathrm{Q}-\mathrm{S}) .{ }^{*} \mathrm{p}<0.05$ and ${ }^{* *} \mathrm{p}<0.01$. Data were expressed as mean \pm SEM ( $n=15$ per group).

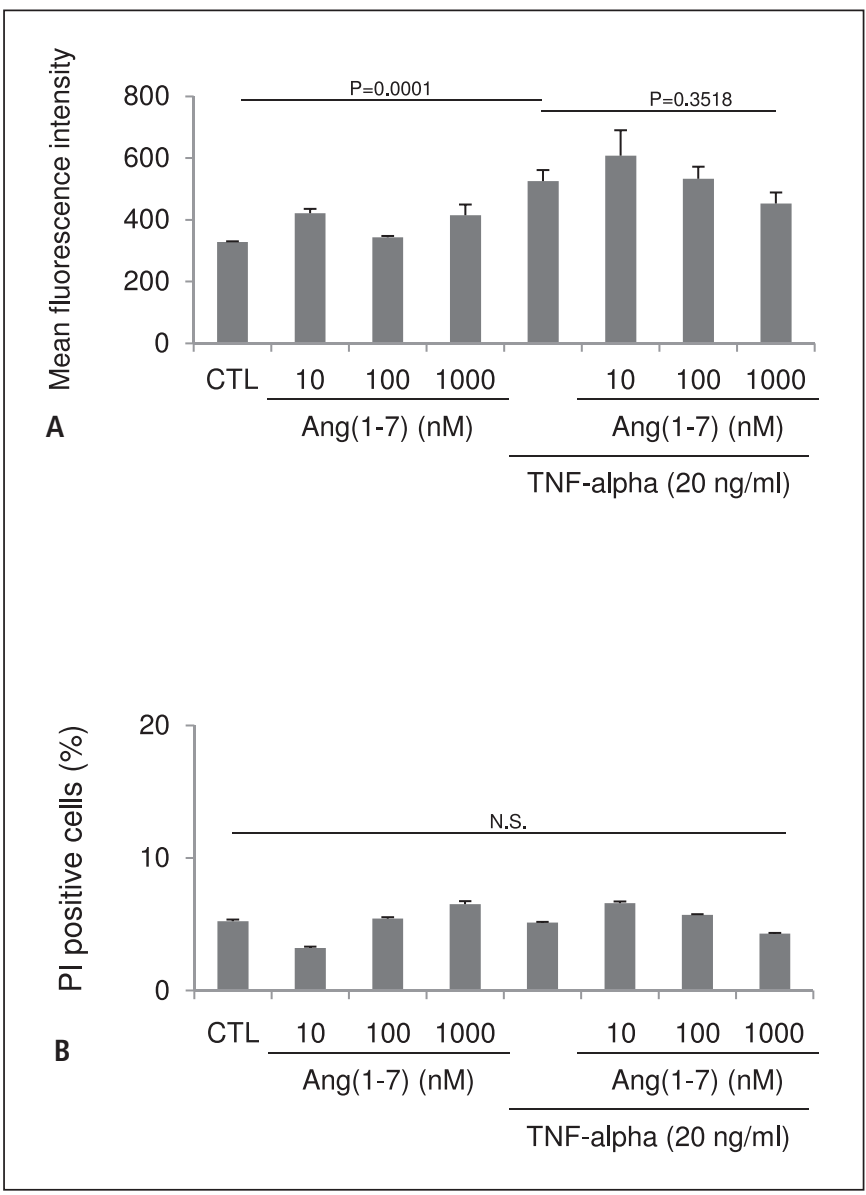

Figure 5: Treatment with Ang-(1-7) does not affect adhesion molecule expression and apoptosis in HECV. Cells were incubated for $3 \mathrm{~h}$ with control medium (CTL), the indicated concentrations of Ang-(1-7) with or without $20 \mathrm{ng} / \mathrm{ml}$ TNF-alpha. Then, flow cytometry analysis of ICAM-1 and $\mathrm{PI}$ on HECV was performed. A) Data are expressed as mean \pm SEM of mean fluorescence intensity $(n=9-13)$. B) Data are expressed as mean \pm SEM of percentages of PI-positive cells $(n=3)$. n.s.: non-significant.

tained increase in the Ang-(1-7) serum levels (27, 42). Since this peptide has a short plasmatic half-life of 10-15 sec (46), this characteristic is essential for the action of the Ang-(1-7). Cyclodextrin inclusion compounds are largely used by the pharmaceutical industry, since they promote enhancement of drug stability, absorption across biological barriers and gastric protection against digestive enzyme degradation $(42,47)$. It has been suggest that the Ang(1-7)-CyD inclusion compound passes intact through the gastrointestinal tract until it entirely reaches the colon; in sequence, the colon-microflora breaks the cyclodextrin into small saccharides, delivering the free form of the peptide which is absorbed (27, 47). Thus, only the free form of Ang-(1-7) is absorbed. In the present study, we did not address the pharmacokinetics of Ang-(1-7)CyD; however, previous studies showed that the increase in Ang-(1-7) plasma levels by Ang-(1-7)-CyD sustains for $24 \mathrm{~h}$ (48) and produced actions dependent on the activation of receptor Mas (27). Moreover, studies using the same dosage and posology of the 


\section{What is known about this topic?}

- Carotid atherosclerotic plaque inflammation and vulnerability is influenced by both systemic and local inflammation.

- Serum and intraplaque inflammatory biomarkers have been investigated as potential targets to reduce plaque vulnerability.

\section{What does this paper add?}

- The Mas receptor (a new component of the renin-angiotensin system) is up regulated in human stable carotid plaques.

- Treatment with the Mas receptor agonist Angiotensin (1-7) reduces atherosclerotic inflammation in mouse plaques.

present study showed effective actions in different pathological conditions $(35,36)$. Therefore, our results may shed some lights on the use of Ang-(1-7)-CyD a new pharmacological approach for potentially stabilising atherosclerotic plaques.

\section{Conflicts of interest}

None declared.

\section{References}

1. Yusuf S, Reddy S, Ounpuu S, et al. Global burden of cardiovascular diseases: Part i: General considerations, the epidemiologic transition, risk factors, and impact of urbanization. Circulation 2001; 104: 2746-2753.

2. Kolodgie FD, Gold HK, Burke AP, et al. Intraplaque hemorrhage and progression of coronary atheroma. N Engl J Med 2003; 349: 2316-2325.

3. Waxman S, Ishibashi F, Muller JE. Detection and treatment of vulnerable plaques and vulnerable patients: Novel approaches to prevention of coronary events. Circulation 2006; 114: 2390-2311.

4. Cheng C, Tempel D, van Haperen R, et al. Atherosclerotic lesion size and vulnerability are determined by patterns of fluid shear stress. Circulation 2006; 113 : 2744-2753.

5. Chatzizisis YS, Jonas M, Coskun AU, et al. Prediction of the localization of highrisk coronary atherosclerotic plaques on the basis of low endothelial shear stress: An intravascular ultrasound and histopathology natural history study. Circulation 2008; 117: 993-1002.

6. Koskinas KC, Chatzizisis YS, Baker AB, et al. The role of low endothelial shear stress in the conversion of atherosclerotic lesions from stable to unstable plaque. Curr Opin Cardiol 2009; 24: 580-590.

7. Weiss D, Kools JJ, Taylor WR. Angiotensin ii-induced hypertension accelerates the development of atherosclerosis in apoe-deficient mice. Circulation 2001; 103: 448-454.

8. Mazzolai L, Duchosal MA, Korber M, et al. Endogenous angiotensin II induces atherosclerotic plaque vulnerability and elicits a th1 response in apoe-/- mice. Hypertension 2004; 44: 277-282.

9. Keidar S, Attias J, Heinrich R, et al. Angiotensin ii atherogenicity in apolipoprotein e deficient mice is associated with increased cellular cholesterol biosynthesis. Atherosclerosis 1999; 146: 249-257.

10. Ni W, Kitamoto S, Ishibashi M, et al. Monocyte chemoattractant protein-1 is an essential inflammatory mediator in angiotensin ii-induced progression of established atherosclerosis in hypercholesterolemic mice. Arterioscler Thromb Vasc Biol 2004; 24: 534-539.

11. Victorino GP, Newton CR, Curran B. Effect of angiotensin ii on microvascular permeability. J Surg Res 2002; 104: 77-81.

12. Keidar S, Heinrich R, Kaplan M, et al. Angiotensin ii administration to atherosclerotic mice increases macrophage uptake of oxidized ldl: A possible role for interleukin-6. Arterioscler Thromb Vasc Biol 2001; 21: 1464-1469.
13. Osada-Oka M, Kita H, Yagi S, et al. Angiotensin at1 receptor blockers suppress oxidized low-density lipoprotein-derived formation of foam cells. Eur J Pharmacol 2012; 679: 9-15.

14. Nickenig G, Harrison DG. The AT(1)-type angiotensin receptor in oxidative stress and atherogenesis: Part ii: AT(1) receptor regulation. Circulation 2002; 105: 530-536.

15. Hernandez-Presa M, Bustos C, Ortego M, et al. Angiotensin-converting enzyme inhibition prevents arterial nuclear factor-kappa $\mathrm{b}$ activation, monocyte chemoattractant protein-1 expression, and macrophage infiltration in a rabbit model of early accelerated atherosclerosis. Circulation 1997; 95: 1532-1541.

16. Tham DM, Martin-McNulty B, Wang YX, et al. Angiotensin ii is associated with activation of nf-kappab-mediated genes and downregulation of ppars. Physiol Genomics 2002; 11: 21-30.

17. Ishibashi M, Hiasa K, Zhao Q, et al. Critical role of monocyte chemoattractant protein-1 receptor ccr2 on monocytes in hypertension-induced vascular inflammation and remodeling. Circ Res 2004; 94: 1203-1210.

18. Bader M. Tissue renin-angiotensin-aldosterone systems: Targets for pharmacological therapy. Annu Rev Pharmacol Toxicol 2010; 50: 439-465.

19. Ferreira AJ, Murca TM, Fraga-Silva RA, et al. New cardiovascular and pulmonary therapeutic strategies based on the angiotensin-converting enzyme 2/angiotensin-(1-7)/mas receptor axis. Int J Hypertens 2012; 2012: 147825.

20. Ferreira AJ, Santos RA. Cardiovascular actions of angiotensin-(1-7). Braz J Med Biol Res 2005; 38: 499-507.

21. Ferreira AJ, Santos RA, Bradford CN, et al. Therapeutic implications of the vasoprotective axis of the renin-angiotensin system in cardiovascular diseases. Hypertension 2010; 55: 207-213.

22. Santos RA, Simoes e Silva AC, Maric C, et al. Angiotensin-(1-7) is an endogenous ligand for the g protein-coupled receptor mas. Proc Natl Acad Sci USA 2003; 100: 8258-8263.

23. Tesanovic S, Vinh A, Gaspari TA, et al. Vasoprotective and atheroprotective effects of angiotensin (1-7) in apolipoprotein e-deficient mice. Arterioscler Thromb Vasc Biol 2010; 30: 1606-1613.

24. Jawien J, Toton-Zuranska J, Gajda M, et al. Angiotensin-(1-7) receptor mas agonist ameliorates progress of atherosclerosis in apoe-knockout mice. J Physiol Pharmacol 2012; 63: 77-85.

25. Wang Y, Tikellis C, Thomas MC, Golledge J. Angiotensin converting enzyme 2 and atherosclerosis. Atherosclerosis 2013; 226: 3-8.

26. Lula I, Denadai AL, Resende JM, et al. Study of angiotensin-(1-7) vasoactive peptide and its beta-cyclodextrin inclusion complexes: Complete sequence-specific nmr assignments and structural studies. Peptides 2007; 28: 2199-2210.

27. Fraga-Silva RA, Costa-Fraga FP, De Sousa FB, et al. An orally active formulation of angiotensin-(1-7) produces an antithrombotic effect. Clinics 2011; 66: 837-841.

28. Montecucco F, Lenglet S, Gayet-Ageron A, et al. Systemic and intraplaque mediators of inflammation are increased in patients symptomatic for ischaemic stroke. Stroke 2010; 41: 1394-1404.

29. Randomised trial of endarterectomy for recently symptomatic carotid stenosis: final results of the MRC European Carotid Surgery Trial (ECST). Lancet 1998; 351: 1379-1387.

30. Barnett HJ, Taylor DW, Eliasziw M, et al. Benefit of carotid endarterectomy in patients with symptomatic moderate or severe stenosis. North american symptomatic carotid endarterectomy trial collaborators. N Engl J Med 1998; 339: 1415-1425.

31. Halliday A, Mansfield A, Marro J, et al. Prevention of disabling and fatal strokes by successful carotid endarterectomy in patients without recent neurological symptoms: Randomised controlled trial. Lancet 2004; 363: 1491-1502.

32. Montecucco F, Di Marzo V, da Silva RF, et al. The activation of the cannabinoid receptor type 2 reduces neutrophilic protease-mediated vulnerability in atherosclerotic plaques. Eur Heart J 2012; 33: 846-856.

33. Olivon VC, Fraga-Silva RA, Segers D, et al. Arginase inhibition prevents the low shear stress-induced development of vulnerable atherosclerotic plaques in apoe-/- mice. Atherosclerosis 2013; 227: 236-243.

34. Montecucco F, Vuilleumier N, Pagano S, et al. Anti-apolipoprotein a-1 autoantibodies are active mediators of atherosclerotic plaque vulnerability. Eur Heart J 2011; 32: 412-421.

35. Feltenberger JD, Andrade JM, Paraiso A, et al. Oral formulation of angiotensin-(1-7) improves lipid metabolism and prevents high-fat diet-induced hepatic steatosis and inflammation in mice. Hypertension 2013; 62: 324-330. 
36. Marques FD, Ferreira AJ, Sinisterra RD, et al. An oral formulation of angiotensin-(1-7) produces cardioprotective effects in infarcted and isoproterenoltreated rats. Hypertension 2011; 57: 477-483.

37. Ramkhelawon B, Vilar J, Rivas D, et al. Shear stress regulates angiotensin type 1 receptor expression in endothelial cells. Circ Res 2009; 105: 869-875.

38. Vickers C, Hales P, Kaushik V, et al. Hydrolysis of biological peptides by human angiotensin-converting enzyme-related carboxypeptidase. J Biol Chem 2002; 277: 14838-14843.

39. Zisman LS, Meixell GE, Bristow MR, Canver CC. Angiotensin-(1-7) formation in the intact human heart: In vivo dependence on angiotensin ii as substrate. Circulation 2003; 108: 1679-1681.

40. Campbell DJ, Zeitz CJ, Esler MD, et al. Evidence against a major role for angiotensin converting enzyme-related carboxypeptidase (ace2) in angiotensin peptide metabolism in the human coronary circulation. J Hypertens 2004; 22: 1971-1976.

41. Sluimer JC, Gasc JM, Hamming I, et al. Angiotensin-converting enzyme 2 (ace2) expression and activity in human carotid atherosclerotic lesions. J Patho 2008; 215: 273-279.

42. Fraga-Silva RA, Ferreira AJ, Dos Santos RA. Opportunities for targeting the angiotensin-converting enzyme 2/angiotensin-(1-7)/mas receptor pathway in hypertension. Curr Hypertens Rep 2013; 15: 31-38.
43. Caro CG, Fitz-Gerald JM, Schroter RC. Arterial wall shear and distribution of early atheroma in man. Nature 1969; 223: 1159-1160.

44. Dai G, Kaazempur-Mofrad MR, Natarajan S, et al. Distinct endothelial phenotypes evoked by arterial waveforms derived from atherosclerosis-susceptible and -resistant regions of human vasculature. Proc Natl Acad Sci USA 2004; 101: 14871-14876.

45. Dong B, Zhang C, Feng JB, et al. Overexpression of ace 2 enhances plaque stability in a rabbit model of atherosclerosis. Arterioscler Thromb Vasc Biol 2008; 28: 1270-1276.

46. Yamada K, Iyer SN, Chappell MC, Ganten D, Ferrario CM. Converting enzyme determines plasma clearance of angiotensin-(1-7). Hypertension 1998; 32: 496-502.

47. Uekama K. Design and evaluation of cyclodextrin-based drug formulation. Chem Pharm Bull 2004; 52: 900-915.

48. World Intellectual Property Organization, Process of preparation of formulations of the peptide angiotensin-(1-7) and its analogues, agonistic and antagonists using cyclodextrins, lipossomes and biodegradable polymers and/or mixtures and products thereof Available at: http://patentscope.wipo.int/search/en/ WO2003039434. Accessed October 18, 2013.

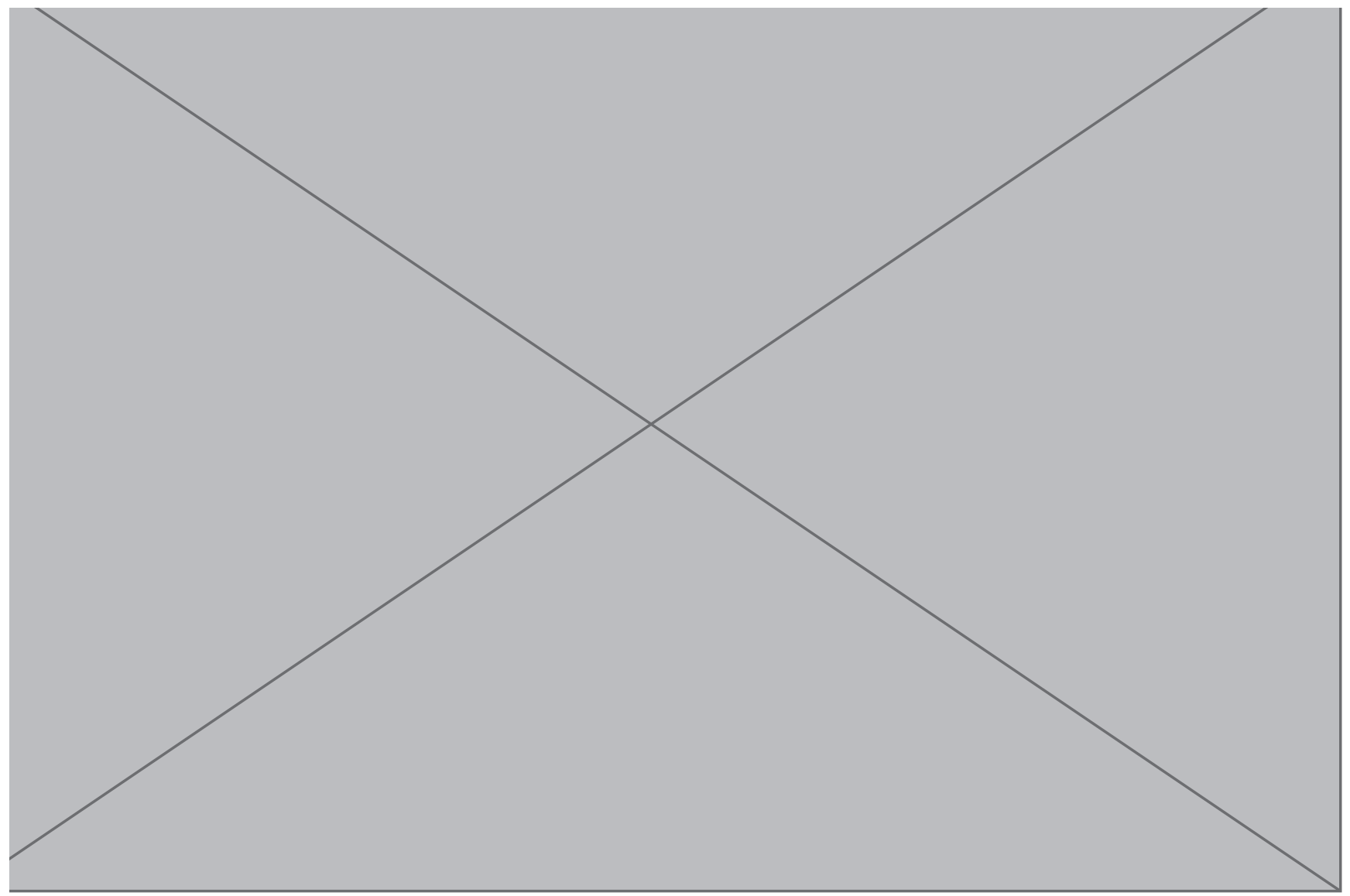

